The distinctions they make are illustrated with the help of sounding records, bottom profiles, charts and underwater photographs, and, where possible, with the results of coring and dredging, magnetic and gravity anomalies, seismic soundings and heat-flow measurements.

Among the most remarkable features are the abyssal plains, where the underlying topography has been smoothed over by sediment-laden currents so that only the highest hills and seamounts stick out like islands from the surface of a lake. Mid-ocean canyons are steep-walled, flat-floored channels, 15 miles wide and perhaps 100 fathoms deep, cut in abyssal plains, generally in the approaches to steps to neighbouring, lower, plains. The mid-ocean ridge is about 1,200 miles wide with varied and irregular topography, and having a median rift, the rift valley, which, so far as we can see, is continuous down the length of the ridge.

Their new ideas are presented carefully, and, while giving much new information and many new ideas, the authors show where they need further support, and what we ought to do next. The book is a special paper of the Geological Society of America. Like several of the Society's publications, it will do much to further the study of the sea. It should be useful to cable companies, because of the new light it throws on some of the natural hazards and destructive processes that occur on the sea-floor. It has an excellent bibliography.

G. E. R. DEACON

\section{SECTOR-FOCUSED CYCLOTRONS}

\section{Sector-Focused Cyclotrons}

Proceedings of an Informal Conference, Sea Island, Georgia, February 2-4, 1959. Edited by F. T. Howard. (Nuclear Science Series-Report No. 26. Publication No. 656.) Pp. xii + 291. (Washington, D.C.: National Academy of Sciences-National Research Council, 1959.) 2.50 dollars.

A CCELERATOR development has progressed with two main objectives in view: the first, to generate higher and higher energies, and the second, to obtain greater intensities. It was in the interests of this latter aim that the Sea Island Conference was held. Scientists from laboratories in America and Europe met to exchange information and ideas on the ways of increasing the intensities of medium- and highenergy cyclotrons by employing sector-focusing. It was fitting that L. H. Thomas, the first to expound this principle in 1938, should be among those present. Having been interested meanwhile in other subjects he could be forgiven his surprise at the delayed-action effect of his work.

The application of frequency modulation to cyclotrons in 1946 effectively solved the problem of obtaining higher energies but only at the expense of intensity, the continuous beam of the conventional eyclotron being sacrificed for a pulsed beam having a duty cycle unattractive for many nuclear experiments. For a time the attractions of the higher energies quenched the desire for greater intensity until of recent years its pursuit has been revived in several laboratories.

The Proceedings of the Conference record the papers and discussions at six sessions, the first of which was devoted to theoretical studies and orbit calculations. In this work, the use of large computers had proved invaluable. The achieving of the required magnetic field, however, was generally by way of successive approximations on an experiment model initially and later at full scale. Various methods of magnetic field measurement were described, many of which were mechanized to reduce the amount of repetitive work.

In the session devoted to radio-frequency problems novel approaches had been made in order to obtain a wide tuning range to give variable energy facilities. Then followed a session on quality of the beam, when interesting results were reported from several laboratories. This led naturally to problems of beamextraction in which resonance phenomena, either deliberately induced or inherent in ridge-focusing, were discussed. The final session included a variety of topics relevant to experiments on cyclotrons, not the least important of which was that of shielding.

Approximately fifty papers were presented and the attendance of more than 80 is indicative of the wide interest in this field of work. Since the Conference several of the accelerators mentioned have come into operation. Perhaps these successes were in some measure helped forward by useful information exchanged there and certainly future workers will benefit from its recording in this series.

M. SNOWDEN

\section{THE PROBLEM OF RETIREMENT}

\section{Growing Old in a Mechanized World}

The Human Problem of a Technical Revolution. By F. Le Gros Clark. (Studies of Ageing within the Conditions of Modern Industry.) Pp. 147. (London: The Nuffield Foundation, 1960.) 7s. 6d. net.

M. F. Le GROS CLARK, with the same clear 1 insight into the urgent practical problems confronting the older worker, restates his theme and provides us with additional data. What is happening to and what is likely to happen to the working man between the ages of 60 and 75 , if he remains in good general hoalth, wishes to continue in employment, but because of declining powers finds it more and more difficult to hold his own in the conditions of modern industry ? What effect is the progressive trend towards mechanization and automation likely to have on the prospects of the ageing man ? To provide some data towards an answer to these questions, Mr. Le Gros Clark takes eight technologically advanced industries (motor-cars, rayon yarn, plate-glass, etc.) and examines the position of the older worker in sample factories.

His conclusions are fairly pessimistic-more pessimistic than those of his earlier reports, also published by the Nuffield Foundation. The accelerating progress of mechanization and automation is beginning to affect the prospects of the older employee. Not only is automatized work more demanding in itself (in the need for vigilance, paced performance, etc.) but also the range of alternative 'light' jobs to which older workers might have been transferred is becoming progressively limited. A rigidly imposed retiring age is making it increasingly difficult for the fit man of 65 or more to remain in employment. We must expect to find more and more men retired from manufacturing industry while they are still reasonably healthy. Are these men likely to find alternative work, if they wish to, in other fields of employment ? In a long appendix Mr. Le Gros Clark considers the older man's prospects in other sorts of work 